Discussion Paper No. 1026

\title{
A MANUFACTURER'S INCENTIVE TO OPEN ITS DIRECT CHANNEL AND ITS IMPACT ON WELFARE
}

\author{
Noriaki Matsushima \\ Tomomichi Mizuno \\ Cong Pan
}

March 2018

The Institute of Social and Economic Research

Osaka University

6-1 Mihogaoka, Ibaraki, Osaka 567-0047, Japan 


\title{
A manufacturer's incentive to open its direct channel and its impact on welfare*
}

\author{
Noriaki Matsushima ${ }^{\dagger}$ Tomomichi Mizuno $\$$ Cong Pan ${ }^{\S}$
}

March 27, 2018

\begin{abstract}
We consider a bilateral monopoly in which a manufacturer can open its direct channel that is less efficient than the existing retailer. We find the following results. The manufacturer opens its direct channel if its bargaining power over the existing retailer is weak. Opening the direct channel is detrimental to social welfare if this channel is efficient. Under a linear demand specification, if the equilibrium unit price under such opening is higher than that under no opening, the opening reduces social welfare under most of the parameter range of the efficiency of the manufacturer's direct channel.
\end{abstract}

Keywords: distribution channels, supplier encroachment, two-part tariff contract, welfare

JEL Classification Numbers: L14, L22, M11, D43

${ }^{*}$ We thank the seminar participants at Shinshu University for their valuable discussions and comments. We gratefully acknowledge the financial support from the Japan Society for the Promotion of Science (JSPS), KAKENHI Grant Numbers JP15H03349, JP15H05728, JP17H00984, and JP16K17116. The usual disclaimer applies.

$\dagger$ Institute of Social and Economic Research, Osaka University, Mihogaoka 6-1, Ibaraki, Osaka, 567-0047, Japan. Phone: (81)-6-6879-8571. Fax: (81)-6-6879-8583. E-mail: nmatsush@iser.osaka-u.ac.jp

${ }^{\ddagger}$ Graduate School of Economics, Kobe University, 2-1 Rokkodai, Nada, Kobe, Hyogo, 657-8501, Japan. Phone: (81)-78-803-6802. Fax: (81)-78-803-7289. E-mail: mizuno@econ.kobe-u.ac.jp

${ }^{\S}$ Faulty of Economics, Nagoya University of Commerce \& Business (NUCB), 4-4 Sagamine Komenokicho Nisshin-shi, Aichi, 470-0193, Japan. Phone: (81)-561-73-2111. Fax: (81)-561-73-1202.

E-mail: pancongecon@gmail.com 


\section{Introduction}

Manufacturers often open their own direct channels to expand accessibility to consumers even when they indirectly sell products through traditional retail channels. Owing to the tougher downstream competition in those markets, such introductions of direct channels, known as "encroachment," intuitively seem welfare-improving, although existing retailers that trade with those manufacturers fall into difficulties to maintain profits as large as before. Such a positive effect of encroachment on welfare seems more likely to hold if a manufacturer is efficient. This fact has put policymakers in a dilemma of whether encroachment should be given policy support from the perspective of social welfare or legally restrained to protect existing retailers' benefits (Kalnins, 2004). ${ }^{1}$

Because of the common expectation of a positive impact of encroachment on welfare (Dutta et al., 1999; Blair and Lafontaine, 2005), the welfare magnitude of this important issue has not been theoretically considered in the economics literature, except for the recent study by Pan (2018), who considers an ex ante downstream duopoly wherein two retailers are offered take-it-or-leave-it contracts secretly from a monopoly manufacturer. ${ }^{2}$ The main focus of Pan (2018) is to show that encroachment may result in higher price and lower consumer surplus although the negative impact of encroachment on social welfare is also discussed in his concluding remarks. A manufacturer using encroachment to solve its commitment problem plays a key role in Pan (2018), meaning that the counterintuitive result is driven by two important elements: (i) the ex ante downstream duopoly of existing retail channels and (ii) contract secrecy. Specifically, in Pan (2018), encroachment may reduce

\footnotetext{
${ }^{1}$ Some theoretical studies even show that such an introduction of a direct channel does not always harm existing retailers (see Arya et al., 2007; Matsushima and Mizuno, 2018). These studies provide theoretical support for the positive effect of encroachment on existing retailers.

${ }^{2}$ This market structure is extensively discussed in the literature on supplier opportunism. The main finding is that under such a structure, the upstream monopolist faces a commitment problem in that it fails to achieve a monopoly outcome (e.g., Hart and Tirole, 1990; McAfee and Schwartz, 1994; Reisinger and Tarantino, 2015).
} 
social welfare because it changes the market outcomes from an ex ante duopoly to an ex post quasi-monopoly. ${ }^{3}$ Moreover, he does not discuss the impact of encroachment on existing retailers because their ex ante and ex post profits are always zero owing to take-it-or-leave-it offers.

In the current study, we consider a manufacturer-retailer (bilateral monopoly) relation so that the manufacturer's commitment problem is no longer a concern. A bargaining problem is also considered so that the pros and cons of encroachment can be tracked from the perspectives of the players. Further, the ex ante market status is a bilateral monopoly in our study, implying that our result is motivated by a different and new mechanism than in Pan (2018).

We consider a bilateral monopoly in which a manufacturer can open its direct channel, which is less efficient than the existing retailer. We need to compare two cases: (i) the manufacturer does not open its direct channel and (ii) it opens its direct channel, inducing a downstream duopoly. In the first case, the manufacturer and retailer determine a two-part tariff contract through Nash bargaining, inducing them to set the unit price at the marginal production cost of the manufacturer in equilibrium. In the second case, they also determine a two-part tariff contract through Nash bargaining by considering the joint profits including the profit of the manufacturer's direct channel. This consideration distorts the unit price in equilibrium because the trading pair needs to balance the quantities in the direct and indirect channels by controlling the unit price that directly influences the quantity of the existing retailer.

We first show that the manufacturer opens its direct channel if its bargaining power over the existing retailer is weak. We then show that the distortion through opening the manufacturer's direct channel is detrimental to social welfare even if the direct channel is efficient.

\footnotetext{
${ }^{3}$ We call the ex post status a quasi-monopoly because it is exactly a monopoly only when selling directly is as efficient as selling via existing retailers.
} 
This is because the trading pair would then overly depend on the production of the manufacturer's direct channel, which is still less efficient than the existing retailer. The welfare property is a novelty of our study. In addition, under a linear demand setting, if the equilibrium unit price under encroachment is higher than that under no encroachment, opening a direct channel reduces social welfare and the existing retailer's profit under most of the parameter range of the efficiency of the manufacturer's direct channel. This outcome has an important policy implication that the competition authority should consider a claim by an existing retailer that trades with an encroaching manufacturer if the claim is based on an increase in its unit price, which is another novelty of our study.

Another closely related study is Reisinger and Tarantino (2015) whose ex ante market structure is close to that in Pan (2018). A monopoly manufacturer that secretly supplies two competing retailers with asymmetric marginal costs in a take-it-or-leave-it manner (downstream duopoly) faces a commitment problem. The authors study the manufacturer's incentive for vertical integration in solving this problem. The main finding is that when the manufacturer chooses to integrate with the inefficient retailer, it will subsidize the other efficient retailer to optimally reallocate the channel distribution. ${ }^{4}$ This finding implies that vertical integration is welfare-improving (welfare-reducing) if and only if the degree of subsidization is high (low). This setting is similar to ours in that encroachment can also be comprehended as downward integration with an inactive retailer. However, in Reisinger and Tarantino (2015), integration with the inefficient retailer rather than the efficient one is always suboptimal for the manufacturer, implying that the welfare-reducing impact of vertical integration can only happen off the equilibrium path. ${ }^{5}$ Conversely, our study offers a new insight that the welfare-reducing downward entry may actually happen in equilibrium.

The remainder of the paper is as follows. Section 2 provides the model setting. Section

\footnotetext{
${ }^{4}$ The Nash bargaining setting is also considered in the web appendix of Reisinger and Tarantino (2015).

5 As an extension of their model, by incorporating marginal cost uncertainty, they show that both downstream retailers can be chosen by the manufacturer as a partner for vertical integration.
} 
3 shows the analytical outcome of the model. Section 4 presents the welfare property of the outcome in Section 3. Section 5 concludes.

\section{Model}

Let us first consider a monopoly supply chain that comprises one upstream manufacturer $U$ and one downstream retailer $D$. $U$ supplies final products to $D$ that then resells them to consumers. $U$ can also choose whether to directly supply to consumers through a direct channel, which is known as "supplier encroachment." We assume that $D$ incurs no cost in the reselling process. On the contrary, when $U$ encroaches, it incurs a positive marginal cost for retailing $c .{ }^{6}$ For simplicity, $U$ 's production cost is normalized to zero.

The trading term between $U$ and $D$ is determined through a negotiation over a two-part tariff contract comprising a unit price $w$ and a fixed fee $f$. The negotiation outcome is decided by the Nash bargaining solution. The bargaining power of $U$ over $D$ is $\beta \in(0,1)$. We assume that $U$ 's direct channel and $D$ supply homogeneous final products in the retail market. ${ }^{7}$ Denote $D$ 's quantity by $q_{D}$ and $U$ 's by $q_{U}$ (if it encroaches). We assume that the inverse demand function $P(Q)$ for final products is nonnegative, strictly decreasing, and twice differentiable, where $P$ is the price and $Q$ is the total quantity sold in the retail market. To guarantee that profit functions are strictly quasi-concave and that resale competition involves strategic substitutability, we assume $P^{\prime}(Q)+Q P^{\prime \prime}(Q)<0$ (Vives, 1999).

The game proceeds as follows. In stage $1, U$ chooses whether to encroach. In stage $2, U$

${ }^{6}$ The assumption that retailers are more efficient than manufacturers is common in the literature. Such an efficiency gap occurs for various reasons. For example, in competition between bricks-and-mortar retailers and manufacturers' online stores, the latter are less familiar with consumers' preferences than the former, which benefit from direct contact (Arya et al., 2007). Moreover, the latter incur higher transportation costs by shipping directly to consumers, whereas the former benefit from bulk shipping ( $\mathrm{Li}$ et al., 2015). Further, the latter must risk returns and redress because consumers cannot physically inspect products before ordering (Pan, 2016).

${ }^{7}$ If they compete in heterogeneous products, $U$ would have a stronger incentive to encroach because it would enjoy an market expansion effect by doing so. 
and $D$ negotiate over the two-part tariff contract. In stage 3 , if $U$ encroached in stage $1, D$ and $U$ simultaneously set their own quantities; otherwise, only $D$ sets its own quantity.

The timeline in which $U$ 's encroaching decision comes before the contracting process follows the idea that starting a direct channel is relatively irreversible and thus must be taken prudently. For example, to conduct direct sales, whether through an online store or a physical direct store, $U$ has to deal with resale issues such as inventory and siting locations, which are always regarded as long-term decisions.

\section{Analysis}

The game is solved by backward induction. Based on $U$ 's decision in stage 1 , there are two types of subgame: $U$ encroaches or not. We use the superscripts $e$ and $n$ to denote each subgame. Note that the Nash bargaining process naturally guarantees that the negotiation between $U$ and $D$ succeeds in equilibrium and that $U$ does not foreclose $D$ because $D$ is more efficient than $U$ 's direct channel.

\subsection{U does not encroach}

First, we discuss the subgame wherein $U$ does not encroach. In stage 3, given the unit price assigned in stage $2, D$ sets quantity $q$ to maximize its profit:

$$
q(w) \equiv \underset{q}{\arg \max }(P(q)-w) q .
$$

To simplify the notation, I define the industry profit as $\Pi^{M}(w) \equiv P(q(w)) q(w)$, where the superscript $M$ represents the integrated monopoly. ${ }^{8}$ Anticipating the outcome in stage 3, $U$

\footnotetext{
${ }^{8}$ In this case, $U$ and $D$ act as if they are integrated as one agent. They jointly solve their maximization problem and then divide the aggregate profit based on their bargaining powers. The payments via the unit price become internal transfers and thus do not affect the industry profit.
} 
and $D$ know that if the negotiation succeeds, they can obtain

$$
\pi_{U}^{n}=w q(w)+f, \quad \pi_{D}^{n}=(P(q(w))-w) q(w)-f .
$$

On the contrary, if the negotiation breaks down, both of them obtain zero profits. The negotiation in stage 2 specifies the contract as follows:

$$
\max _{w, f}\left\{\pi_{U}^{n}\right\}^{\beta}\left\{\pi_{D}^{n}\right\}^{1-\beta}
$$

The first-order condition can be denoted as follows:

$$
\begin{aligned}
& \frac{\partial q}{\partial w} w=0 \\
\Rightarrow \quad & w^{n}=0 ; f^{n}=\beta \Pi^{M}(0) .
\end{aligned}
$$

The corresponding profits of $U$ and $D$ are

$$
\pi_{U}^{n}=\beta \Pi^{M}(0) ; \quad \pi_{D}^{n}=(1-\beta) \Pi^{M}(0)
$$

This result is standard. With a two-part tariff contract, $U$ always sets the unit price to its production cost (zero) and abstracts $D$ 's surplus through the fixed fee based on its bargaining power.

\section{2 $U$ encroaches}

Next, let us consider the subgame wherein $U$ encroaches. In this case, it sells through both $D$ and its direct channel. The following maximization problems in stage 3 are

$$
\max _{q_{D}}\left(P\left(q_{D}, q_{U}\right)-w\right) q_{D}-f, \max _{q_{U}}\left(P\left(q_{D}, q_{U}\right)-c\right) q_{U}+q_{D} w+f
$$


leading to the subgame quantities: $q_{D}(w, c)$ and $q_{U}(w, c)$. We define the industry profit as follows (we use the superscript $I$ to represent it):

$$
\Pi^{I}(w, c) \equiv P\left(q_{D}(w, c), q_{U}(w, c)\right) q_{D}(w, c)+\left[P\left(q_{D}(w, c), q_{U}(w, c)\right)-c\right] q_{U}(w, c)
$$

If the negotiation succeeds, they can obtain

$$
\begin{aligned}
& \pi_{U}^{e}(w, c, f)=\left[P\left(q_{D}(w, c), q_{U}(w, c)\right)-c\right] q_{U}(w, c)+w q_{D}(w, c)+f \\
& \pi_{D}^{e}(w, c, f)=\left[P\left(q_{D}(w, c), q_{U}(w, c)\right)-w\right] q_{D}(w, c)-f .
\end{aligned}
$$

On the contrary, if the negotiation breaks down, $U$ has a disagreement payoff in which it directly sells and monopolizes the retail market with marginal cost $c$, although $D$ gains zero profit. The profits of $U$ and $D$ in the negotiation breakdown are given as

$$
\pi_{U}^{e \prime}=\Pi^{M}(c), \pi_{D}^{e \prime}=0
$$

The bargaining problem in stage 2 is given as

$$
\max _{w, f}\left\{\pi_{U}^{e}(w, c, f)-\pi_{U}^{e \prime}\right\}^{\beta}\left\{\pi_{D}^{e}(w, c, f)\right\}^{1-\beta}
$$

leading to

$$
\begin{aligned}
& w^{e} \equiv \underset{w}{\arg \max } \Pi^{I}(w, c)-\Pi^{M}(c), \\
& f^{e}=(1-\beta)\left[\Pi^{M}(c)-\left(P\left(q_{D}\left(w^{e}, c\right)+q_{U}\left(w^{e}, c\right)\right)-c\right) q_{U}\left(w^{e}, c\right)\right] \\
& \quad+\beta\left[P\left(q_{D}\left(w^{e}, c\right)+q_{U}\left(w^{e}, c\right)\right) q_{D}\left(w^{e}, c\right)-w^{e} q_{D}\left(w^{e}, c\right)\right] .
\end{aligned}
$$


Owing to the bargaining procedure, they set $w$ as if they maximize their joint profit through the control of $w$ and split the maximized joint profit through fixed fee $f$. By using the envelop theorem, we derive the first-order condition of $w$ :

$$
\frac{\partial q_{D}}{\partial w} w+\underbrace{P^{\prime}(\cdot) \frac{\partial q_{D}}{\partial w} q_{U}+P^{\prime}(\cdot) \frac{\partial q_{U}}{\partial w} q_{D}}_{\text {commitment of encroachment }}=0 .
$$

In addition to the first term of Eq. (1) in the case without encroachment, the second and third terms are included. Those terms reflect the control of the downstream quantities through $w$. Specifically, the second term of Eq. (2) denotes a positive effect on $w$, while the third term denotes a negative one. Intuitively, as marginal cost $c$ increases, the relative efficiency of $D$ improves, inducing the bargaining pair to increase $q_{D}$ through a decrease in $w$. In other words, we expect that the positive correlation between $c$ and $q_{D}$ is monotonic. Here, we show that the statement actually holds true.

We remark on the equilibrium property in the second- and third-stage outcomes. D's quantity $q_{D}$ is ultimately controlled by unit price $w$, which implies that the two-part tariff contract can be regarded as a quantity-based one, $\left(q_{D}, f\right)$. We can convert the procedure in the second and third stages as follows: the bargaining in stage 2 is that $U$ chooses $q_{D}$ to maximize the joint profit of the bargaining pair, anticipating $q_{U}\left(q_{D}\right)$, which will be chosen by $U$ 's direct channel in stage 3 . In stage 3, because $U$ has already levied the fees on $D$ in stage 2, it ignores the impact on $D$ 's profit. In other words, $U$ solves the following:

$$
\max _{q_{U}}\left[P\left(q_{D}, q_{U}\right)-c\right] q_{U}
$$

from which we have $U$ 's best-response function $q_{U}\left(q_{D}, c\right)$. In stage $2, U$ solves the following:

$$
\max _{q_{D}}\left[P\left(q_{D}, q_{U}\left(q_{D}, c\right)\right)-c\right] q_{U}\left(q_{D}, c\right)+P\left(q_{D}, q_{U}\left(q_{D}, c\right)\right) q_{D}-\Pi^{M}(c)
$$


Lemma 1 The optimal $q_{D}$ is given by

$$
q_{D}^{e}=-c \times \frac{\left(P^{\prime \prime} q_{U}+2 P^{\prime}\right)}{\left(P^{\prime}\right)^{2}}(>0) .
$$

Proof. The first-order condition of $U$ 's direct channel in stage 3 is given by

$$
P^{\prime} q_{U}+P-c=0
$$

Totally differentiating Eq. (5) gives rise to

$$
\frac{d q_{U}}{d q_{D}}=-\frac{P^{\prime \prime} q_{U}+P^{\prime}}{P^{\prime \prime} q_{U}+2 P^{\prime}} .
$$

By using Eq. (6), the maximization problem in Eq. (3) can be derived as

$$
\begin{aligned}
& {\left[P^{\prime} q_{U}+P-c\right] \frac{d q_{U}}{d q_{D}}+P^{\prime} q_{D} \frac{d q_{U}}{d q_{D}}+P^{\prime} q_{U}+P^{\prime} q_{D}+P=0 } \\
\Rightarrow & P^{\prime} q_{D} \frac{d q_{U}}{d q_{D}}+c+P^{\prime} q_{D}=0 \\
\Rightarrow & q_{D}=\frac{-c}{P^{\prime} \times\left(1+d q_{U} / d q_{D}\right)} .
\end{aligned}
$$

Substituting Eq. (6) into the last equation gives rise to the expression in Lemma 1. Because of strategic substitutability, $q_{D}$ is positive.

Lemma 1 implies that as long as $D$ has a cost advantage, it is always assigned a positive share proportional to $c$ by $U$. Because of continuity, it is straightforward that when $c$ is almost zero, $q_{D}^{e}$ will be close to zero. Owing to strategic substitutability, $q_{U}^{e}$ will be close to the monopoly quantity under which its marginal cost is zero. Then, the positive effect in Eq. (2) becomes a dominant one so that $w^{e}>0$. This fact is summarized by the following lemma. 
Lemma $2 \exists \bar{c}>0$ such that $\forall c<\bar{c}, w^{e}>0$.

$D$ is possibly offered a tax in a bilateral monopoly with a two-part tariff contract. Given that $U$ has committed to encroachment, when its direct channel is efficient, it would rather restrain $D$ 's sales and shift some share back to $U$ 's direct channel.

In Reisinger and Tarantino (2015), when a manufacturer supplies duopoly retailers with asymmetric marginal costs and the manufacturer integrates with the less efficient retailer, the more efficient one will be offered a subsidy. This result is similar but essentially different to ours because we consider the case wherein the manufacturer creates a new retailer (i.e., encroachment) instead of integrating with an incumbent one. In other words, the baseline situation in Reisinger and Tarantino (2015) is an asymmetric downstream duopoly with a monopoly manufacturer, whereas that in our study is a bilateral monopoly with the possibility of supplier encroachment. In our study, the manufacturer's decision on whether to encroach is explicitly considered and the subsidy can exist in the subgame perfect equilibrium. This part is discussed after we derive Proposition 1.

\subsection{U's incentive to encroach}

Note that with a general demand function, we cannot explicitly derive the equilibrium unit price $w^{e}$. Despite this, given a certain $w^{e}$ that satisfies Eq. (2), the corresponding profits in this subgame can be denoted as

$$
\pi_{U}^{e}=\beta \Pi^{I}\left(w^{e}, c\right)+(1-\beta) \Pi^{M}(c) ; \pi_{D}^{e}=(1-\beta)\left[\Pi^{I}\left(w^{e}, c\right)-\Pi^{M}(c)\right]
$$

To restrict our attention to the parameter range wherein encroachment happens in equilibrium, we need to confirm $U$ 's incentive to encroach within the parameter range wherein its direct channel is active. Let $\hat{c}$ such that $q_{U}^{e}>0$ for any $c<\hat{c}$. By comparing $\pi_{U}^{n}$ with $\pi_{U}^{e}$, 
we derive the following equation:

$$
\pi_{U}^{e}-\pi_{U}^{n}=\beta\left[\Pi^{I}\left(w^{e}, c\right)-\Pi^{M}(0)\right]+(1-\beta) \Pi^{M}(c) .
$$

Compared with the monopoly case with zero marginal cost, the industry profit of the duopoly case with one agent having a positive marginal cost (i.e., $c>0$ ) is strictly lower, no matter how $U$ chooses $w^{e}$. In other words, an efficiency loss at the industry level is inevitable. Hence, the first term of Eq. (7) is always negative. On the contrary, when $c<\hat{c}$, the second term must be positive. ${ }^{9}$ Therefore, whether $U$ encroaches is decided by $U$ 's bargaining power, which is summarized in Proposition 1.

Proposition 1 Given that $c<\hat{c}$, $U$ encroaches when its bargaining power is relatively small. Formally,

$$
\beta<\frac{\Pi^{M}(c)}{\Pi^{M}(c)+\Pi^{M}(0)-\Pi^{I}\left(w^{e}, c\right)} .
$$

When $U$ 's bargaining power is weak, the transfer from $D$ in the bilateral monopoly is small. Encroachment enhances $U$ 's bargaining position through an increase in its disagreement payoff, whereas it diminishes the total industry profit.

The threshold value of $\beta$ in Proposition 1 is always located in the interval $(0,1)$. Then, by comparing it with the case of no encroachment, $U$ encroaching may either raise or reduce the unit price contingent on the value of $c$, which contrasts with most of the literature on supplier encroachment. ${ }^{10}$

\footnotetext{
${ }^{9} \Pi^{M}(c)$ is positive if the monopoly price is higher than $c$. The condition $c<\hat{c}$ guarantees that $q_{U}^{e}$ is positive in duopoly competition and thus that the duopoly price is higher than $c$. Because the monopoly price is always higher than the duopoly price, $\Pi^{M}(c)$ must be positive if $c<\hat{c}$ is satisfied.

${ }^{10}$ In all studies modeling a linear contract, the unit price must reduce after encroachment (e.g., Arya et al., 2007); by contrast, in all research that models a two-part tariff contract, the unit price must increase after encroachment (e.g., Matsushima and Mizuno, 2018; Pan, 2018).
} 


\section{Welfare}

We check the impact of $U$ 's encroachment on the total surplus and consumer surplus. First, we consider it under the general demand function. Second, by employing a linear demand function, we explicitly solve the problem in the previous section.

The social surplus is denoted by

$$
W=\int_{0}^{q_{U}\left(q_{D}^{e}(c), c\right)+q_{D}^{e}(c)} P(x) d x-c q_{U}\left(q_{D}^{e}(c), c\right) .
$$

By differentiating $W$ with respect to $c$, we have

$$
\frac{d W}{d c}=P\left(q_{U}\left(q_{D}^{e}(c), c\right)+q_{D}^{e}(c)\right) \frac{d\left(q_{U}\left(q_{D}^{e}(c), c\right)+q_{D}^{e}(c)\right)}{d c}-q_{U}\left(q_{D}^{e}(c), c\right)-c \times\left(q_{U}\left(q_{D}^{e}(c), c\right)\right)^{\prime}
$$

At $c=0$, the differential is

$$
\left.\frac{d W}{d c}\right|_{c=0}=\left.P\left(q^{M}\right) \frac{d\left(q_{U}\left(q_{D}^{e}(c), c\right)+q_{D}^{e}(c)\right)}{d c}\right|_{c=0}-q^{M},
$$

where $q^{M}$ is the monopoly quantity in which its marginal cost is zero. From Eqs. (4), (5), and (6), we have the following relation: ${ }^{11}$

$$
\begin{aligned}
\left.\frac{d\left(q_{U}\left(q_{D}^{e}(c), c\right)+q_{D}^{e}(c)\right)}{d c}\right|_{c=0} & =\left.\left(d q_{U} / d q_{D}+1\right) \frac{d q_{D}^{e}(c)}{d c}\right|_{c=0}+\left.\frac{\partial q_{U}}{\partial c}\right|_{c=0} \\
& =-\frac{P^{\prime}}{P^{\prime \prime} q^{M}+2 P^{\prime}} \times \frac{\left(P^{\prime \prime} q^{M}+2 P^{\prime}\right)}{\left(P^{\prime}\right)^{2}}+\frac{1}{P^{\prime \prime} q^{M}+2 P^{\prime}} \\
& =-\frac{P^{\prime \prime} q^{M}+P^{\prime}}{P^{\prime}\left(P^{\prime \prime} q^{M}+2 P^{\prime}\right)}>0 .
\end{aligned}
$$

\footnotetext{
${ }^{11}$ By substituting Eq. (6) into the first line of Eq. (9), we obtain the first fraction in the second line of Eq. (9). By simply differentiating $q_{D}^{e}$ in Eq. (4) with respect to $c$, we obtain the second fraction in the second line of Eq. (9). Finally, from the partial derivative of $q_{U}$ in Eq. (5) with respect to $c$, we obtain $\partial q_{U} / \partial c$ at $c=0$.
} 
By using the above outcomes in Eqs. (8) and (9) and Eq. (5) at $c=0$, we obtain

$$
\left.\frac{d W}{d c}\right|_{c=0}=-P \frac{P^{\prime \prime} q^{M}+P^{\prime}}{P^{\prime}\left(P^{\prime \prime} q^{M}+2 P^{\prime}\right)}-q^{M}=\frac{P}{P^{\prime \prime} q^{M}+2 P^{\prime}}<0
$$

Note that the social surplus under no encroachment is the same as that under encroachment when $c=0$. In addition, the consumer surplus under no encroachment is the same as that under encroachment when $c=0$. From the facts and results in Eqs. (9) and (10), we have Proposition 2.

Proposition 2 For small c, U's encroachment is detrimental to the social surplus, but beneficial to the consumer surplus.

When $c$ is small, the market share of $U$ 's direct channel is large, implying that the relatively inefficient channel handles most retailing. This inefficient allocation of production worsens the social surplus.

Linear demand case Second, we set a linear inverse demand function, $p=a-b Q$, to further investigate the welfare property of the model. The other settings are the same as in the main model: the profits are $\pi_{U}=\left[a-b\left(q_{U}+q_{D}\right)-c\right] q_{U}+w q_{D}+f$ and $\pi_{D}=$ $\left[a-b\left(q_{U}+q_{D}\right)-w\right] q_{D}-f$, while the Nash product is $N P=\left[\pi_{U}-O P\right]^{\beta}\left[\pi_{D}\right]^{1-\beta}$, where $O P=(a-c)^{2} /(4 b)$ if $U$ encroaches and $O P=0$ otherwise.

We first consider the case without encroachment. In the third stage, from the first-order conditions $\partial \pi_{D} / \partial q_{D}=0$ and $q_{U}=0$, we have $q_{D}^{L n}(w)=(a-w) /(2 b)$, where the superscript Ln denotes the case with no encroachment under linear demand. By substituting this result into $N P$ and solving the first-order conditions $\partial N P / \partial w=0$ and $\partial N P / \partial f=0$ for $w$ and $f$, we have $w^{L n}=0$ and $f^{L n}=a^{2} \beta /(4 b)$. From the above results, we obtain the outcomes as 
follows:

$$
\begin{aligned}
& q_{U}^{L n}=0, \quad q_{D}^{L n}=\frac{a}{2 b}, w^{L n}=0, \quad f^{L n}=\frac{a^{2} \beta}{4 b}, \\
& \pi_{U}^{L n}=\frac{a^{2} \beta}{4 b}, \quad \pi_{D}^{L n}=\frac{a^{2}(1-\beta)}{4 b}, C S^{L n}=\frac{a^{2}}{8 b}, \quad W^{L n}=\frac{3 a^{2}}{8 b} .
\end{aligned}
$$

Next, we consider the case with encroachment. In the third stage, from the first-order conditions $\partial \pi_{D} / \partial q_{D}=0$ and $\partial \pi_{U} / \partial q_{U}=0$, we have $q_{U}^{L e}(w)=(a-2 c+w) /(3 b)$ and $q_{D}^{L e}(w)=(a-2 w+c) /(3 b)$, where the superscript Le denotes the case with encroachment under linear demand. By substituting this result into each firm's profit, we have $\pi_{U}^{L e}(w)$ and $\pi_{D}^{L e}(w)$. In the second stage, we derive the optimal two-part tariff. Since the outside option with encroachment does not depend on $w$ and $f$, we can calculate the optimal contract as follows. The first step is to maximize the net joint profit, $\pi_{U}^{L e}(w)-(a-c)^{2} /(4 b)+\pi_{D}^{L e}(w)$, with respect to $w$. Then, we have $w^{L e}=(a-5 c) / 2$. The second step is to divide the maximized net joint profit by $f$. Then, by solving $\pi_{D}^{L e}\left(w^{L e}\right)=(1-\beta)\left[\pi_{U}^{L e}\left(w^{L e}\right)-(a-c)^{2} /(4 b)+\pi_{D}^{L e}\left(w^{L e}\right)\right]$ for $f$, we have $f^{L e}=c^{2}(3+\beta) / b$. From the above results, we obtain the outcomes as follows:

$$
\begin{aligned}
& q_{U}^{L e}=\frac{a-3 c}{2 b}, \quad q_{D}^{L e}=\frac{2 c}{b}, w^{L e}=\frac{a-5 c}{2}, f^{L e}=\frac{c^{2}(3+\beta)}{b}, \\
& \pi_{U}^{L e}=\frac{(a-c)^{2}+4 c^{2} \beta}{4 b}, \pi_{D}^{L e}=\frac{c^{2}(1-\beta)}{b}, \quad C S^{L e}=\frac{(a+c)^{2}}{8 b}, \quad W^{L e}=\frac{3 a^{2}-2 a c+11 c^{2}}{8 b} .
\end{aligned}
$$

From these outcomes, we can show that Lemmas 1 and 2 are satisfied. In other words, for any $c>0, q_{D}^{L e}>0$; and for any $c<a / 5, w^{L e}>0$.

In the first stage, $U$ decides to encroach if $\pi_{U}^{L e}>\pi_{U}^{L n}$. By solving this for $\beta$, we have

$$
\beta<\frac{(a-c)^{2}}{(a-2 c)(a+2 c)} .
$$

This condition corresponds to Proposition 1.

Finally, we compare the social surplus with the consumer surplus in the two cases. From 
$C S^{L n}$ and $W^{L n}$ in Eq. (11) and $C S^{L e}$ and $W^{L e}$ in Eq. (12), we have Proposition 3.

Proposition 3 Under linear demand, the social surplus under encroachment is lower than that under no encroachment if and only if $0<c<2 a / 11$. In addition, the consumer surplus under encroachment is always larger than that under no encroachment.

The former argument in Proposition 3 confirms that a welfare reduction caused by encroachment is more likely to occur when the direct channel is sufficiently efficient. The latter argument follows from the fact that $C S^{L e}$ is increasing in $c$. This is similar to that in Pan and Yoshida (2018), who consider an international oligopoly wherein foreign manufacturers carrying out FDI sell products through local retailers and foreign-made products directly through e-commerce sites. ${ }^{12}$

We also check how encroachment affects the profit of $D$ and the unit price, namely we check $\pi_{D}^{L e}-\pi_{D}^{L n}$ and $w^{L e}-w^{L n}$. The calculations lead to the following proposition, which confirms Lemma 2.

Proposition 4 Encroachment always decreases the profit of D. It increases the unit price if and only if $c<a / 5$.

The latter argument in Proposition 4 is almost consistent with the condition in Proposition 3 that encroachment is detrimental to the social surplus. This finding implies that an increase in $w$ can be a signal that encroachment is welfare-reducing and that the competition authority should consider a claim by an existing retailer that trades with an encroaching manufacturer if the claim is based on an increase in its unit price.

\footnotetext{
${ }^{12}$ In their model, local bilateral supply chains also supply local products. The model structure is similar to ours in that the cost disadvantage in direct selling is captured by a specific tariff in Pan and Yoshida (2018). The main purpose of Pan and Yoshida (2018) is to show that a tariff reduction may result in a higher local price, which thus reduces the local consumer surplus. Although the model setting in Pan and Yoshida (2018) partially overlaps that in our study, we focus on comparing welfare with encroachment with that without encroachment. Such a comparison is outside the scope of Pan and Yoshida (2018).
} 


\section{Conclusion}

We consider a bilateral monopoly model in which an upstream manufacturer that trades with a downstream retailer can open its direct channel (so-called supplier encroachment). We show that encroachment by the manufacturer may harm social welfare, although it changes the downstream market from a monopoly to a duopoly. This finding complements the recent study by Pan (2018), who also shows that supplier encroachment may harm social welfare in an ex ante downstream duopoly. Under a linear demand specification, we show that welfare-decreasing encroachment occurs almost along with an increase in the unit price for the downstream retailer, which implies that the competition authority should consider a claim by an existing retailer that trades with an encroaching manufacturer if the claim is based on an increase in its unit price.

Matsushima and Mizuno (2018) extend the linear demand setting by incorporating the cost-reducing efforts of an existing retailer. The main concern of Matsushima and Mizuno (2018), however, is how the threat of supplier encroachment influences the retailer's effort level and economic welfare. Our study and Matsushima and Mizuno (2018) thus complement each other.

\section{References}

[1] Arya, A.; Mittendorf, B. and Sappington, D. E. M., 2007, The bright side of supplier encroachment. Marketing Science 26(5), 651-659.

[2] Blair, R. D. and Lafontaine, F., 2005, The Economics of Franchising. Cambridge, UK: Cambridge University Press.

[3] Dutta, S.; Heide, J. and Bergen, M., 1999, Vertical territory restrictions and public policy: Theories and industry evidence. Journal of Marketing 63(4), 121-134. 
[4] Hart, O. and Tirole, J., 1990, Vertical integration and market foreclosure. Brookings Papers on Economic Activity: Microeconomics 1990, 205-276.

[5] Kalnins, A., 2004, An empirical analysis of territorial encroachment within franchised and company-owned branded chains. Management Science 23(4), 1-20.

[6] Li, Z.; Gilbert, S. M. and Lai, G., 2015, Supplier encroachment as an enhancement or a hindrance to nonlinear pricing. Production and Operations Management 24(1), 89-109.

[7] Matsushima, N. and Mizuno, T., 2018, Supplier encroachment and retailer effort. ISER Discussion Paper No. 1027. Osaka University.

[8] McAfee, R. P. and Schwartz, M., 1994, Opportunism in multilateral vertical contracting: Nondiscrimination, exclusivity and uniformity. American Economic Review 84(1), 210-230.

[9] Pan, C., 2016, Retailer's product line choice with manufacturer's multichannel marketing. ISER Discussion Paper No. 938. Osaka University.

Available at SSRN: http://ssrn.com/abstract=2808837.

[10] Pan, C., 2018, Supplier encroachment and consumer welfare: Upstream firm's opportunism and multichannel distribution. ISER Discussion Paper No. 1020. Osaka University.

Available at SSRN: https://ssrn.com/abstract=3124026.

[11] Pan C. and Yoshida, S., 2018, Multichannel distribution under international oligopoly. Mimeo.

[12] Reisinger, M. and Tarantino, E., 2015, Vertical integration, foreclosure, and productive efficiency. RAND Journal of Economics 46(3), 461-479. 
[13] Vives, X. 1999. Oligopoly Pricing. Cambridge, Massachusetts: MIT Press. 